\title{
Recent results on GPD/DVCS experiments at CLAS
}

\author{
Jacques Ball * \\ SPhN/Irfu, CEA, Centre de Saclay \\ 91191 Gif sur Yvette, France \\ E-mail: Jacques.Ballecea.fr
}

\section{The CLAS Collaboration}

Thomas Jefferson National Accelerator Facility

Newport-News, Virginia, USA

URL: http://WwW.JLab.org/Hall-B/

Generalized Parton Distributions (GPDs) are the new powerful means to describe the nucleon. The related deeply exclusive experiments have started since the beginning of this century mainly the study of Deeply Virtual Scattering processes. Experiments running at Jefferson Laboratory, on the CEBAF Large Acceptance Spectrometer (CLAS) in Hall B, have used both polarized and unpolarized targets and beams. Data analysis is working out beam spin and target spin asymmetries as well as cross-sections. Recent results are presented and comparison with theory display the foreseen difficulties to be encountered when trying to extract the GPDs from the observables. 


\section{Introduction}

The electromagnetic probe has been, for many decades, the tool of choice to analyze the nucleon. The sophistication of the achieved experiments has followed closely the improvement of the technology needed to produce high precision beams while the reachable energies increased over the years. The nucleon, which was the basic studied object by accessing its inner components, quarks and gluons, has become a metalaboratory in itself. In parallel to the instrumentation progress, new theoretical tools have been developed to understand the nucleon structure at deeper and deeper levels. While elastic electron scattering enabled to measure nucleon form factors which reflect the spatial shape of charge distributions, deep inelastic scattering experiments (DIS) measured parton distribution functions which determine longitudinal momentum and helicity distributions. The Generalized Parton Distributions (GPDs) formalism includes both former approaches and carry more information about the dynamical degrees of freedom inside the nucleon. GPDs [1-4] enable to access the spatial distribution of quarks and gluons inside the nucleon and also the quark orbital momentum which is one of the keys to solve the nucleon spin issue. For hard processes, the factorization is a way to separate the hard scattering part of the interaction, which is calculable in pertubative $\mathrm{QCD}$, and the nonpertubative nucleon structure part parametrized through GPDs. In particular this applies to Deeply Virtual Compton Scattering (DVCS) [5] in the Björken regime, at high $Q^{2}$, which is today the obvious process to access experimentally GPDs.

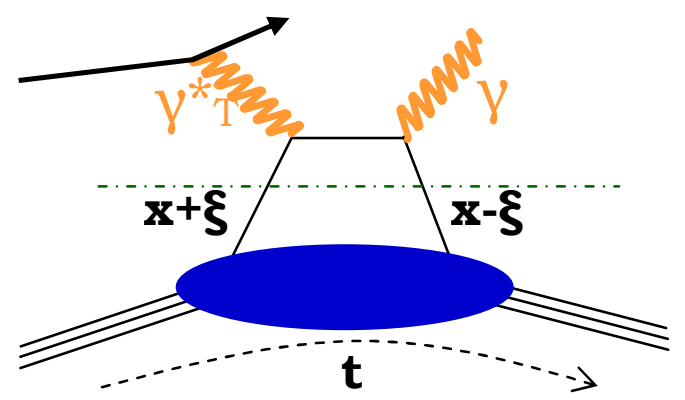

Figure 1 : DVCS diagram, also known as "handbag model", where $x$ is the average longitudinal momentum fraction of the hit quark in the initial and final states, $t$ is the squared four-momentum transfer and $\xi$ the skewedness related to the Björken variable $\left(\xi \approx x_{B} /\left(2-x_{B}\right)\right.$.

In the DVCS process (see Fig. 1), electron scattering on the nucleon occurs via a virtual photon $\gamma^{*}$ which hits one quark off the nucleon, a real photon $\gamma$ is emitted and the quark is reinjected in the nucleon. The incoming and outgoing electrons are characterized by their respective momenta $p$ and $p^{\prime}$, while $q$ and $q^{\prime}$ are the momenta related to the photons. The amplitude for removing a quark with momentum fraction $x+\xi$ and restoring it with momentum fraction $x-\xi$ is related to $H, \tilde{H}, E$ and $\tilde{E}$ the proton four chiral even GPDs. These GPDs depend on kinematical variables $x, t=\left(p-p^{\prime}\right)^{2}, Q^{2}=-\left(k-k^{\prime}\right)^{2}$, and $\xi$. Observables are integrals in $x$ of GPDs.DVCS is not distinguishable, experimentally, from the Bethe Heitler process $(\mathrm{BH})$ occuring simultaneously where the outgoing photon is emitted wether by the initial or the scattered electron.In the kinematic region of the CLAS experiments $\left(Q^{2}\right.$ from 1 to 
$4.8 \mathrm{GeV}^{2}, x_{B}$ from 0.11 to 0.58 , $-t$ from 0.09 to $1.8 \mathrm{GeV}^{2}$ ), the $\mathrm{BH}$ cross section is dominant but the interference between the two amplitudes enhances the DVCS contribution. The measured cross-section can be written as [6]:

$$
\frac{d^{4} \sigma}{d x_{B} d Q^{2} d t d \varphi} \approx\left|T_{B H}\right|^{2}+\left|T_{D V C S}\right|^{2}+2 T_{B H} \cdot \operatorname{Re}\left(T_{D V C S}\right)
$$

With $\varphi$ defined as the angle between the leptonic and hadronic planes, $T_{D V C S}$ and $T_{B H}$ as the DVCS and BH amplitudes.

Using a polarized electron beam on an unpolarized target the cross-sections differences for two opposite helicities can be expressed as:

$$
\frac{d^{4} \vec{\sigma}-d^{4} \bar{\sigma}}{d x_{B} d Q^{2} d t d \varphi} \approx 2 T_{B H} \cdot \operatorname{Im}\left(T_{D V C S}\right)+\left[\left|T_{D \vec{V} C S}\right|^{2}-\left|T_{D \bar{V} C S}\right|^{2}\right]
$$

The $\left|T_{D V C S}\right|^{2}$ amplitudes are small and can be neglected and the BH amplitude can be calculated, so that equations (1) and (2) give access to the real and imaginary part of the DVCS amplitudes.It is a fact that asymmetries are more accessible to measurement than cross section differences. The first evidences to test the validity of the handbag model came from beam spin asymmetries which were expected to behave with a $\sin \varphi$ dependance [7]. DVCS enables to measure observables which are related to a combination of the four GPDs $H, \tilde{H}, E, \tilde{E}$. The polarization is indeed the way to disentangle the GPDs. Using a polarized electron beam on an unpolarized target boosts GPD $H$, unpolarized beam on a longitudinally polarized target enables access to $\tilde{H}$, and on transversally polarized target to $E[3,8]$. The extraction of GPDs from observables requires a deconvolution and a large set of data. A global fit analysis might lead to a model independent extraction of the GPDs.

\section{Results from CLAS}

\subsection{The experimental set up}

Beam spin asymmetries and target spin asymmetries have been measured in Jefferson Lab Hall B through two dedicated DVCS experiments e1-DVCS ${ }^{1}$ and eg1-DVCS ${ }^{2}$ respectively. After a first running period in 2005, the e1-DVCS experiment took data again between the end of 2008 and the beginning of 2009 while eg1-DVCS had been running in three parts during 2009. Except for the targets, both experiments used the same set-up of the CLAS spectrometer [9]. The regular CLAS has a toroidal magnet based on six superconducting coils set symmetrically around the beam line. The available space between the coils is filled with identical packages of detectors, consisting of three regions of Drift Chambers (DC) and one set of Scintillator Counters (SC). They cover an angle range of 8 to $140^{\circ}$ and are used for the tracking and the particle identification. These equipments are completed in the forward direction by Čerenkov Counters (CC) and Electromagnetic calorimeters (EC) for electron identification; the $\mathrm{EC}$ is also used to identify and detect photons and neutrons. 
The DVCS exclusive experiments had to detect the three particles in the final state of the reaction $\vec{e} p \rightarrow e p \gamma$. Therefore a new Inner Calorimeter (IC), made of $424 \mathrm{PbWO}_{4}$ crystals, covering a polar angular range of 5 to $15^{\circ}$ was added to detect photons in the energy range of 1 to $5 \mathrm{GeV}$. Moreover, a $4.7 \mathrm{~T}$ superconducting solenoid, placed around the target was designed to trap around the beam axis the low energy background Møller electrons produced in the target though enabling the detection of recoil protons up to $60^{\circ}$.

e1-DVCS used a $2.5 \mathrm{~cm}$ long liquid hydrogen target hit by the $5.77 \mathrm{GeV}$ CEBAF polarized electron beam (mean polarization of 79.4\%), yielding a luminosity of $2.10^{34} \mathrm{~cm}^{-2} \mathrm{~s}^{-1}$.

eg1-DVCS is currently running using a $\mathrm{NH}_{3}$ longitudinally polarized target to measure target spin asymmetries as well as double spin asymmetries. The average target polarization is about $80 \%$.

\subsection{Results}

Beam spin asymmetry defined as $A_{L U}=\frac{d^{4} \vec{\sigma}-d^{4} \bar{\sigma}}{d^{4} \vec{\sigma}+d^{4} \bar{\sigma}}$ for +1 and -1 beam helicities, can be derived as: $A_{L U}=\frac{a \sin \varphi}{1+c \cos \varphi+d \cos 2 \varphi}$

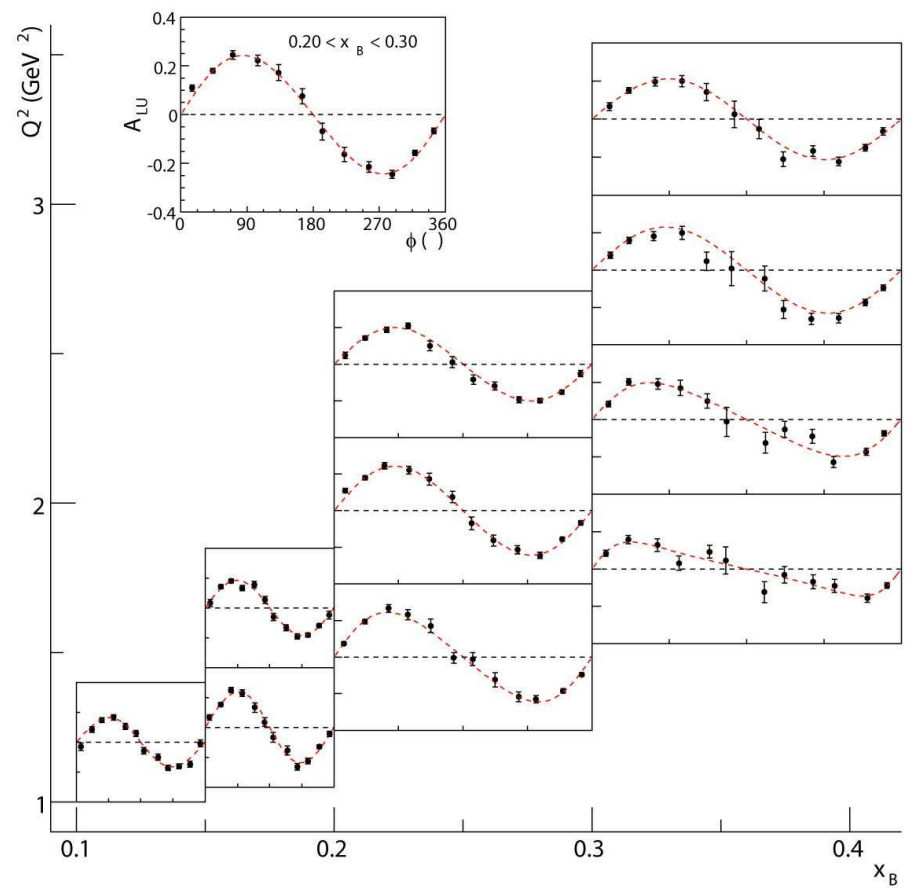

Figure 2: Beam spin asymmetry as a function of $\varphi$ in the $\left(x_{B}, Q^{2}\right)$ space. Black dots are the data, dashed line is a fit using equ(3).

For small values of $|t|$, the GPD $H$ can be considered as dominant and $a, c, d$ are parameters related to the DVCS, BH amplitudes via harmonic coefficients of $\varphi$ [8] and accessible at points $x= \pm \xi$. The conditions of data analysis and yielding of results are 
detailed in [9]. The results of beam spin asymmetries $\varphi$ distributions integrated over $t$ are displayed on fig. 2 and show accurate data in good agreement with a fit based on equ (3) where the contribution of $d$ had been neglected as it is supposed to be consistent with 0 . At $90^{\circ}$, $A_{L U}$ reduces to $a$ and is displayed on Fig. 3 compared with theoretical models.

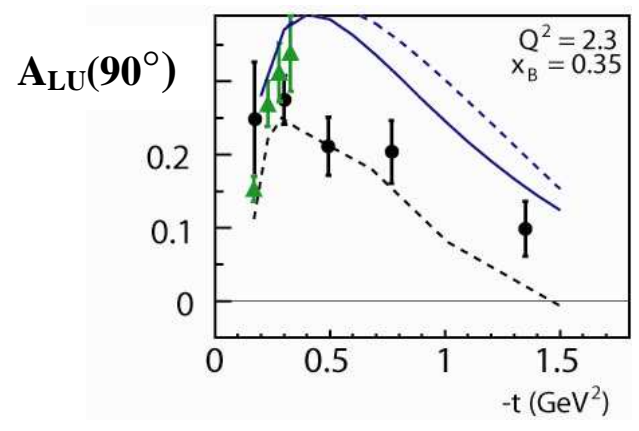

Figure 3: $A_{L U}\left(90^{\circ}\right)$ as a function of -t for a particular bin in $Q^{2}$ and $x_{B}$. Data are black dots, triangles are extracted from cross section measurements in JLab Hall A [11], lower dashed line comes from a Regge inspired model[12], solid and upper dashed line correspond to GPDs calculations at twist 2 and twist 3 order respectively [3].

The double distribution models [3] which incorporate GPDs mathematical properties (polyniomality of the Mellin moments) and have elastic form factors and parton distribution functions as limiting values overestimate the data and the twist 3 contribution is even in larger disagreement. A Regge inspired model [12] in which DVCS is considered as $\rho$ production followed by $\rho-\gamma$ coupling in the nucleon field or in the vacuum is in good agreement with the data for $-t \leq 1 G e V^{2}$.

\subsection{Future results}

With the ending of the second part of experiment e1-DVCS, more data on beam spin symmetries will come and soon the first results of cross section measurements will be available. The eg1-DVCS experiment which has already accumulated an equivalent charge of $14 \mathrm{mC}$ and is scheduled to run for 60 days will provide target spin asymmetries which will be very valuable to check the dominance of the GPD $H$.

Theoretical work has recently taken advantage of the incoming of data to improve the known models, see for example [13,14]. It comes out that the discrepancy observed between the data and the existing models, Double Distribution models or $H$ only models, will not decrease significantly even with higher statistics. Sizeable off forward contribution is suggested, dominance of GPD $H$ less obvious, and contribution of higher twist needs to be included in the models. In parallel other approaches using quark soliton models, MIT bag model are still considered.

\section{Conclusion}

The DVCS experiments have started to produce accurate data. The global analyses using sets of measurements in different configurations of beam and target polarization will become more reliable with the incoming amount of new measurements channels as meson deeply virtual meson production or DVCS on the nucleus. Some theoretical hypotheses about the dominance 
of a particular GPD over the others have to be validated. With the upgrade in energy of Jlab from 6 to $12 \mathrm{GeV}$, a larger kinematical region, at higher $Q^{2}$, will be reachable, moreover challenging experiments using transversally polarised targets have been programmed and it may become feasible to discriminate GPD $E$. Moreover the COMPASS experiment at CERN will soon start its own DVCS program, covering a complementary range of very large $Q^{2}$ and low $x_{B}$.

This work was supported in part by the US Department of Energy contract DOE-AC0506OR23177 under which the Jefferson Science Associates, LLC, operates Jefferson Lab, the National Science Foundation, the French Commissariat à l'Energie Atomique and Centre National de la Recherche Scientifique, the Italian Istituto Nazionale di Fisica Nucleare, the Korean Science and Engineering Foundation, the UK Engineering and Physical Science Research Council.

\section{References}

[1] D. Müller et al., Wave functions, Evolution equations and Evolution kernels from light-ray operators of QCD, Fortschr. Phys. 42 (101) 1994.

[2] A.V. Radyushkin, Non-forward parton distributions, Phys. Rev. D56 (5524) 1997.

[3] K. Goeke, M.V. Polyakov and M. Vanderhaegen, Hard exclusive reactions and the structure of hadrons, Prog. Part. Nucl. Phys. 47 (401) 2001.

[4] X. Ji, Gauge-invariant decomposition of nucleon spin, Phys. Rev. Lett. 78 (610) 1997.

[5] J.C. Collins and A. Freund, Proof of factorization for deeply virtual Compton scattering in QCD, Phys. Rev. D59 (074009) 1999.

[6] M. Diehl et al., Testing the handbag contribution to exclusive virtual Compton scattering, Phys. Lett.B411 (193) 1997.

[7] S. Stepanyan et al., Observation of exclusive deeply virtual Compton scattering in polarized electron beam asymmetry measurements, Phys. Rev. Lett. 87 (182002) 2001.

[8] A.V. Belitsky, D. Müller and A. Kirchner, Theory of deeply virtual Compton scattering on the nucleon, Nucl. Phys. B629 (323) 2002.

[9] B. Mecking et al., The CEBAF large acceptance spectrometer Ring, Nucl. Instr.Meth. A503 (513) 2006.

[10] F.X Girod et al., Measurement of Deeply Virtual Compton Scattering Beam Spin Asymmetries, Phys. Rev. Lett.100 (162002) 2008.

[11] C. Muñoz Camacho et al., Scaling Tests of the Cross Section for Deeply Virtual Compton Scattering, Phys. Rev. Lett. 97 (262002) 2006.

[12] J.M. Laget, Unitarity constraints on deeply virtual Compton scattering, Phys. Rev. C76 (052201) 2007.

[13] H. Moutarde, Extraction of the Compton Form Factor $H$ from recent deeply virtual Compton scattering at Jefferson Lab, Phys. Rev. D79 (094021) 2009.

[14] K.Kumerički and D. Müller, Deeply virtual Compton scattering at small $x_{B}$ and the access to the GPD H, arXiv:0904.0458v1 [hep-ph]. 